\title{
PCOS and hyperandrogenism in type 1 diabetes
}

\author{
Susan Tibuni-Sanders, Shahla Nader \\ Department of Internal Medicine, Endocrine Division, University of Texas at Houston, Houston, USA \\ Email: susan.sanders@uth.tmc.edu
}

Received 17 January 2012; revised 18 February 2012; accepted 5 March 2012

\begin{abstract}
Women with both type 1 diabetes and polycystic ovarian syndrome (PCOS) represent a unique population, with long term implications on fertility and diabetic complications, which need to be addressed. The studies reviewed herein address the increased incidence of PCOS in women with type 1 diabetes. The leading theories suggest that the underlying pathophysiology is related to intensive insulin therapy and resulting ovarian hyperandrogenism. We have searched the literature to determine what studies have been done to address the role of insulin resistance and insulin sensitizers for reduction of hyperandrogenism in these patients. According to the available evidence, we conclude that metformin may have a role in reducing the androgenic picture in type 1 patients with PCOS and insulin resistance.
\end{abstract}

Keywords: Type 1 Diabetes Mellitus; Hyperandrogenism; PCOS; Metformin

\section{INTRODUCTION}

Polycystic ovarian syndrome is one of the most common female endocrinologic disorders. It is currently known to affect up to $10 \%$ of women of reproductive age and is one of the leading causes of female sub fertility [1].

The Rotterdam criteria classifies PCOS as 2 of 3 of the following: oligoovulation or anovulation, polycystic ovaries on ultrasound and clinical or biochemical hyperandrogenism. The syndrome is usually associated with insulin resistance, obesity, and type 2 diabetes. This is in contrast to type 1 diabetes which is characterized by autoimmune destruction of insulin producing beta cells of the pancreas, leading to absolute insulin deficiency. Research suggests that insulin resistance can also occur in many type 1 diabetics. This article focuses on typical type 1 diabetic women who appear to have insulin resistance which in the setting of PCOS.

PCOS and hyperandrogenism have been reported in many patients with type 1 diabetes. This paper is a comprehensive review of the literature concerning the asso- ciation of PCOS and type 1 diabetes. The potential underlying mechanisms and possible therapeutic approaches for type 1 diabetic women with PCOS and hyperandrogenism will be explored. Type 1 diabetics with PCOS have unique reproductive issues. The goal is to help improve awareness and discuss the clinical implications of the coexistence of these disorders in this particular patient population.

\section{PREVALENCE}

Since the 1950's there has been increased awareness of menstrual dysfunction in women with type 1 diabetes. Several studies have shown that women with type 1 diabetes experience a delay in the onset of menarche as well as more frequent menstrual disturbances when compared to non-diabetic siblings or healthy controls. A study by Strotmeyer et al revealed that type 1 diabetes was associated with longer cycle length ( $>31$ days), longer menstruation ( $>6$ days), heavier menstruation, and more reports of any menstrual problem for younger age ranges (for women aged $<29$ years) as compared to control women and biological siblings [2].

Recent small studies have shown an increased prevalence of PCOS and hyperandrogenism in patients with type 1 diabetes, yet there is lack of awareness of the coupling of these disorders among practitioners.

The prevalence of menstrual irregularity and hirsutism has been addressed in several studies. A study by Adcock in 1994 found that menstrual irregularity was more prevalent in type 1 diabetic women than controls (54\% versus $21 \% \mathrm{p}<0.01$ ) [3]. Polycystic morphology on ultrasound was identified in 10/13 (77\%) of these type 1 diabetic patients with menstrual irregularity. The type 1 diabetic women also exhibited lower SHBG (sex hormone binding globulin) levels ( $\mathrm{p}<0.025$ ).

To further evaluate this Escobar-Morreale (2000) completed a retrospective clinical analysis of type 1 women in Madrid Spain using the 1990 NIH (National Institutes of Health) criteria for PCOS (oligoovulation and hyperandrogenism) [4]. During his biochemical and clinical assessment of 85 women with type 1 diabetes he 
found $38 \%$ had hyperandrogenic disorders. There was also an $18.8 \%$ prevalence of PCOS ( $p<0.05$ when compared to the prevalence of control non-diabetic women in Madrid-6.5\%).

Analysis of the clinical and biochemical characteristics of type 1 diabetic women in Escobar-Morreale's study revealed no significant difference in the age of menarche, peri-menarchal insulin dose, mean hemoglobin A1C, or mean daily insulin dose to account for the observed hyperandrogenism. Also, there was no association between body mass index and the presence of hyperandrogenic disorders.

Codner et al. re-evaluated this question in a recent study, using the new Rotterdam criteria for PCOS including polycystic ovarian morphology in a Chilean population [5]. Performing a small cross sectional study of type 1 diabetic women, she found that there was a high frequency of biochemical hyperandrogenism $(28.6 \%$ in type 1 diabetic women compared to $0 \%$ in healthy nondiabetic controls - who had regular menses and no history of hyperandrogenism). Although the controls were deliberately eumenorrheic and had no history of hyperandrogenism, nonetheless PCOS was found in a staggering $40.5 \%$ in type 1 diabetic women compared to $2.6 \%$ in control healthy non-diabetic controls). Biochemical hyperandrogenism was present in $23.8 \%$ of the diabetic versus $7.9 \%$ of the controls. PCOS morphology was found in $54.8 \%$ of the diabetic versus $13.2 \%$ of the controls. Oligomenorrhea was present in $19 \%$ of the diabetic women. The proportion of women using intensive insulin treatment versus standard therapy was higher in those with both polycystic ovarian morphology and PCOS (p < 0.05).

While more large population studies are needed to further verify the prevalence of PCOS in type 1 diabetic women, clinicians caring for women with type 1 diabetes mellitus should be aware of the likely increase in frequency between these two disorders.

\section{IMPACT OF HYPERANDROGENISM ON OUTCOMES IN WOMEN WITH TYPE 1 DIABETES}

What are the implications of hyperandrogenism in type 1 diabetic females? Few studies have been completed to properly answer this question. As there appears to be an increased prevalence of hyperandrogenism and PCOS in this population already at high risk for micro and macrovascular disease, it is reasonable to consider the possible additive effects of the coexistence of the two disorders.

Development of microalbuminuria is a known complication of type 1 diabetes typically due to sub-optimal glycemic control. To answer what the effect of hyperandrogenism is on development of microalbuminuria, Amin et al. performed an analysis of IGF-1 (insulin like growth factor-1), IGF-BP (insulin like growth factor binding protein), testosterone and SHBG levels in type 1 diabetic girls (aged 10 - 20 years) and found that there was notably lower IGF-1 levels and a higher androgen index in patients with microalbuminuria $(\mathrm{p}=0.03)$ [6]. In type 1 girls, testosterone levels were higher in those with microalbuminuria compared to those without, even among those with similar hemoglobin A1C levels. Amin concluded that development of microalbuminuria at puberty might reflect not only poor glycemic control, but also changes in ovarian function in the setting of aggressive insulin therapy. The study suggests that it is the aggressive insulin therapy used to control hyperglycemia which leads to increased weight gain as well as ovarian hyperandrogenism. Perhaps other alternative therapies, such as insulin sensitizers might be explored as options to reduce the progression of these microvasular changes.

Studies have shown that non-diabetic women with PCOS have a greater clustering of cardiac risk factors. Is there a causal link between coronary artery disease and PCOS? The WISE (Women and Ischemia Syndrome Evaluation) study in 2008 published their evaluation of 104 post-menopausal women with a history of PCOS based on the 1990 NIH criteria [7]. They found an increased incidence of angiographic coronary artery disease $(\mathrm{p}=0.04)$ and a reduced 5-year event free survival (78.9\%) for women with PCOS vs. $88.7 \%$ for controls (P $=0.006$ ). The relationship of decreased event free survival for PCOS women was maintained in risk adjusted models controlling for age, body mass index, diabetes mellitus, and angiographic coronary artery disease.

Although the WISE study used current androgen levels in post-menopausal women to document hyperandrogenism, the correlation between increased coronary artery disease and history of irregular menses and current hyperandrogenism is striking. It was suggested that the prolonged hyperandrogenism might be one mechanism for this increased risk. This begs the question, in a type 1 diabetic woman, with pre-existing increased cardiac risk factors, what is the potential additive effect of hyperandrogenism and PCOS on the development of coronary artery disease. Again, future studies in this unique cohort of women are needed to investigate this further.

\section{PATHOPHYSIOLOGICAL MECHANISMS}

The underlying cause of hyperandrogenism in type 1 diabetic women is not clear. There have however been studies which have demonstrated the following in nondiabetic PCOS women: 1) insulin stimulates ovarian steroidogenesis in non-diabetic PCOS women in a manner which is greater than that seen in normal women [8] 2) combined stimulation of LH (luteinizing hormone) 
and insulin at normal concentrations may act synergistically to increase androgen biosynthesis in PCOS ovarian tissue.

\subsection{Insulin and Excess Androgens Crosstalk}

In a study by Nestler et al. 25 PCOS women (without diabetes) were investigated for any causal link between hyperinsulinemia and hyperandrogenism [9]. Nestler hypothesized that hyperinsulinemia lead to increased activity of the ovarian cytochrome P4501 alpha enzyme resulting in hyperandrogenism. The ovarian cytochrome P450c17alpha is a bifunctional enzyme which converts progesterone to 17 alpha hydroxyprogesterone through its 17 alpha hydroxylase activity, and then converts 17alpha hydroxyprogesterone to androstenedione through its 17, 20 lyase activity. Nestler suggested that a hallmark of increased ovarian P450c17 alpha activity is an exaggerated serum 17-alpha hydroxyprogesterone response to stimulation by GNRH (gonadotropin releasing hormone) agonists.

\subsection{Insulin Replacement in Type 1 Diabetic Patients Is Non-Physiologic}

Insulin replacement therapy in patients with type 1 diabetes is non-physiologic. In normal persons without diabetes, endogenous insulin is secreted into the portal venous system and approximately $50 \%$ of this initial amount is removed and degraded by the liver. The insulin then acts at the liver and pancreas (to diminish glucagon release) to suppress gluconeogenesis [10]. The periphery then receives half of the insulin initially secreted by the pancreas. In type 1 diabetics however, insulin is administered subcutaneously (bypassing first pass hepatic metabolism) and in larger doses, as required to decrease hepatic gluconeogenesis, than may be peripherally needed to achieve peripheral glucose uptake. This mismatch results in increased hypoglycemia, increased food intake with weight gain, poor blood glucose regulation, and increased peripheral (including ovarian) insulin levels [11]. This non-physiologic insulin administration may have a role in the pathogenesis of hyperandrogenism and type 1 diabetes.

\section{THERAPEUTIC STRATEGIES}

\subsection{Strategies Based on Crosstalk between Insulin and Excess Androgens}

It has also been demonstrated that any treatment which aims at improving insulin resistance in women with PCOS results in lower androgen levels as shown in the second part of Nestler's study [9]. The women in Nestler's study had 17-hyrdoxyprogesterone levels measured in the follicular phase at baseline as well as after leu- prolide stimulation. They were subsequently given either placebo or metformin for four to eight weeks and their 17-hyroxyprogesterone levels pre and post leuprolide stimulation were evaluated. In addition PCOS women were given an oral glucose tolerance test, and serum insulin levels were measured while fasting and after glucose administration. Results revealed that women who received metformin therapy had decreased cytochrome P450c17alpha activity (as demonstrated by a substantial reduction in serum 17-hydroxyprogesterone in response to leuprolide) and a $44 \%$ decrease in serum free testosterone concentrations. In addition the metformin group also had a reduction in the serum insulin concentration during fasting and after administration of oral glucose tolerance test. These results suggest that it is the hyperinsulinemia associated with PCOS which has a synergistic effect on the cytochrome P450c17 alpha activity leading to androgen biosynthesis.

More recently Baillargeon et al. showed [12] that in non-diabetic PCOS women the administration of diazoxide (which directly suppresses pancreatic insulin secretion), lead to significant reductions in serum total testosterone levels.

Codner et al. has evaluated ovarian function in type 1 diabetic girls compared to age matched controls for the effect of leuprolide on 17-hydroxyprogesterone and androgen levels [13]. Her study revealed higher stimulated testosterone levels and 17-hydroxyprogesterone levels after leuprolide stimulation by the end of puberty in type 1 diabetic girls compared to non-diabetic age and body mass index matched controls. This suggests that similar to the non-diabetic PCOS woman, the ovary is the source of the hyperandrogenism in type 1 diabetic women as well.

Adolescents with type 1 diabetes typically have deterioration in metabolic control during puberty, likely due to insulin resistance. Proposed causes of the deterioration in control include the increase in sex steroids during this period, glucotoxicity associated with non-compliance, and the disturbance of the insulin-like growth factor I/growth hormone axis. In response to the deterioration in metabolic control, the doses of insulin are often increased, which may worsen underlying ovarian hyperandrogenism.

Finally, patients with type 1 diabetes may have a family history of insulin resistance, themselves carrying this genetic burden.

\subsection{Metformin and Thiazolidiones Therapy}

The United Kingdom Prospective Diabetes Study (UKPDS) in 2008 showed us that metformin had a benefit in long term risk reduction for any diabetes related endpoint, such as myocardial infarction or death from any cause especially in overweight patients [14]. What, if any, is 
the effect of metformin and thiazolidiones in patients with type 1 diabetes. More importantly is there any indication for use in patients with type 1 diabetes and PCOS? Patients with PCOS are known to have impaired glucose tolerance. Studies by Dunaif et al. indicated that nontype 1 diabetic women with PCOS are more insulin resistant than are unaffected counterparts matched for body mass index, fat free body mass, and body fat distribution [15].

Among many of its actions, metformin has been thought to increase insulin sensitivity in the liver by inhibiting hepatic gluconeogenesis and hepatic glucose output [16]. It is also thought to decrease fatty acid oxidation, intestinal glucose absorption and increase peripheral insulin sensitivity by enhancing glucose uptake in the muscle [17]. Metformin has been associated with modest reductions in triglycerides, VLDL (very low density lipoprotein), LDL (low density lipoprotein) and CRP (c-reactive protein) levels. In the UKPDS, patients randomized to metformin therapy had a $33 \%$ reduced rate of myocardial infarction, which persisted for ten years after initial randomization [14].

Few studies have focused on the effects of metformin on type 1 diabetics. Indeed there have been no studies in particular on Type 1 diabetic women with PCOS and metformin. Recently a Cochrane review article evaluated the effects of metformin in type 1 diabetic adolescents [18].

Sarnblad et al found that there was no evidence of an increase in insulin sensitivity among type 1 patients (aged 16.9+/1.6 years, average hemoglobin A1C 9.5) on metformin. There was, however, a reduction in mean hemoglobin A1C level in the metformin (500 mg daily titrated up to $1000 \mathrm{mg}$ twice daily) + insulin group $(-0.14 / p=0.01)$ as compared to the insulin + placebo group [19]. The same study used a euglycemic clamp to demonstrate that those patients with the greatest insulin resistance had the most benefit from metformin and showed a decrease in insulin dosage by $10 \%$ when compared to the placebo group.

A second systematic review was done by Vella et al. in 2010 [20]. They performed an analysis of nine randomized studies (covering 192 patient years) on the effects of metformin in type 1 diabetes patients of all ages. They found that metformin was associated with a reduction in insulin dose requirements (6.6 u/day; 95\% confidence interval), but no significant change in hemoglobin A1C or weight loss. The effect of insulin dose reduction was statistically significant $(\mathrm{p}<0.001)$.

Patients with type 1 diabetes do not produce insulin. However, in those with PCOS, we are giving increased doses of insulin, especially during puberty, in order to overcome the effects of insulin resistance and control hepatic gluconeogenesis. Further research is needed to investigate whether the use of metformin or other insulin sensitizers may help to increase insulin sensitivity and decrease overall dose, and in effect lower the androgen levels seen in Type 1 diabetic women with PCOS.

\section{CONCLUSIONS}

Ovarian hyperandrogenism and PCOS are prevalent in adolescent and adult women with type 1 diabetes. Data suggests that this is irrespective of diabetic control, hemoglobin A1C, and body mass index. Investigated mechanisms in non-diabetic women with PCOS have demonstrated a clear association between insulin levels and ovarian hyperandrogenism.

Novel treatments for these type 1 diabetic adolescent women, such as insulin sensitizers, could be considered to decrease the mean insulin dose, particularly during puberty when insulin resistance may exacerbate their diabetic control and androgenic symptoms.

Whether such treatment affects the long term wellbeing of the patient, with regards to renal function and cardiovascular health remains to be determined.

\section{REFERENCES}

[1] Ehrmann, D. (2005) Medical progress: Polycystic ovarian syndrome. The New England Journal of Medicine, 352, 1223-1236. doi:10.1056/NEJMra041536

[2] Strotmeyer, E., Steenkiste, M.S., Foley, S. and Dorman, J. (2003) Menstrual cycle differences between women with type 1 diabetes and women without type 1 diabetes. Diabetes Care, 26, 1016-1021. doi:10.2337/diacare.26.4.1016

[3] Adcock, C.J., Perry, L.A., Lindsell, D.R., Taylor, A.M., Holly, J.M. and Dunger, D.B. (1994) Menstrual irregularities are more common in adolescents with type 1 diabetes: Association with poor glycemic control and weight gain. Diabetic Medicine, 11, 465-470. doi:10.1111/j.1464-5491.1994.tb00307.x

[4] Escobar-Morreale, H.F., Roldan, B., Barrio, R., Alonso, M., Sancho, J., De la Calle, H. and Garcia-Robles, R. (2000) High prevalence of polycystic ovary syndrome and hirsutism in women with type 1 diabetes mellitus. Journal of Experimental and Clinical Medicine, 85, 4182-4187.

[5] Codner, E., Soto, N., Lopez, P., Trejo, L., Avila, A., Eyzaguirre, F.C., Iniguez, G. and Cassorla, F. (2006) Diagnostic criteria for polycystic ovary syndrome and ovarian morphology in women with type 1 diabetes mellitus. Journal of Clinical Endocrinology \& Metabolism, 91, 2250-2256.

[6] Amin, R., Schultz, C., Ong, K., Frystyk, J., Dalton, R.N., Perry, L., Orskov, H. and Dunger, D. (2003) Low IGF-1 and elevated testosterone during puberty in subjects with type 1 diabetes developing microalbuminuria in comparison to normoalbuminuric control subjects: The Oxford Regional Prospective Study. Diabetes Care, 26, 
1456-1461. doi:10.2337/diacare.26.5.1456

[7] Shaw, L., Merz, N., Azziz, R., Stanczyk, F., Sopko, G., Braunstein, G., et al. (2008) Postmenopausal women with a history of irregular menses and elevated androgen measurements at high risk for worsening cardiovascular event-free survival: Results from the National Institutes of Health-National Heart, Lung, and Blood Institute sponsored women's ischemia syndrome evaluation. Journal of Clinical Endocrinology \& Metabolism, 93, 1276-1284.

[8] Bolli, G.B. (2001) Physiological insulin replacement in type 1 diabetes mellitus. Experimental and Clinical Endocrinology \& Diabetes, 109, S317-S332. doi:10.1055/s-2001-18591

[9] Nestler, J. and Jakubowicz, D. (1996) Decreases in ovarian cytochrome P450c17 alpha activity and serum free testosterone after reduction of insulin secretion in polycystic ovary syndrome. The New England Journal of Medicine, 335, 617-623. doi:10.1056/NEJM199608293350902

[10] Longo, D., et al. (2011) Harrison's principles of internal medicine. 18th Edition, McGraw-Hill, New York.

[11] Lebovtiz, H.E. (2010) Adjunct therapy for type 1 diabetes mellitus. Nature Reviews Endocrinology, 6, 326-334. doi:10.1038/nrendo.2010.49

[12] Baillargeon, J.P., Jean-Patrice, M.D. and Carpentier, A. (2007) Role of insulin in the hyperandrogenemia of lean women with polycystic ovary syndrome and normal insulin sensitivity. Fertility and Sterility, 88, 886-893. doi:10.1016/j.fertnstert.2006.12.055

[13] Codner, E., Mook-Kanamori, D., Bazares, R.A., Unanue, N., Sovino, H., Ugarte, F., Avial, A., Iniguez, G. and Cassorla, F. (2005) Ovarian function during puberty in girls with type 1 diabetes mellitus: Response to leuprolide. Jour- nal of Clinical Endocrinology \& Metabolism, 90, 39393945. doi:10.1210/jc.2005-0142

[14] Holman, R., Paul, S., Bethel, A., Matthews, D. and Neil, H.A. (2008) 10-year follow up of intensive glucose control in type 2 diabetes. The New England Journal of Medicine, 359, 1577-1589. doi:10.1056/NEJMoa0806470

[15] Dunaif, A., Segal K.R., Futterweit, W. and Dobrjansky, A. (1989) Profound peripheral insulin resistance, independent of obesity, in polycystic ovary syndrome. Diabetes, 38, 1165-1174. doi:10.2337/diabetes.38.9.1165

[16] Hamilton, J., Cummings, E., Zdravkovic, V., Finegood, D. and Daneman, D. (2003) Metformin as an adjunctive therapy in adolescents with type 1 diabetes and insulin resistance. Diabetes Care, 26,138-143. doi:10.2337/diacare.26.1.138

[17] Hermann, L.S. and Melander, A. (1997) Biguanides: Basic aspects and clinical uses. In: Herman, L.S., Ed., International Textbook of Diabetes Mellitus, Wiley John \& Sons Inc., Chichester, 841-864.

[18] Abdelghaffar, S. and Attia, A.M. (2009) Metformin added to insulin therapy for type 1 diabetes mellitus in adolescents. Cochrane Database of Systematic Reviews, CD006691. doi:10.1002/14651858.CD006691.pub2

[19] Sarnblad, S., Kroon, M. and Aman, J. (2003) Metformin as additional therapy in adolescents with poorly controlled type 1 diabetes: Randomized placebo-controlled trial with aspects on insulin sensitivity. European Journal of Endocrinology, 149, 323-329. doi:10.1530/eje.0.1490323

[20] Vella, S., Buetow, L., Ryle, P., Lvingstone, S., Colhoun, H.M. and Petrie, J.R. (2010) The use of metformin in type 1 diabetes: A systematic review of efficacy. Diabetologia, 53, 809-820. doi:10.1007/s00125-009-1636-9 